\title{
Joy of Scanning with Electron and X-ray Imaging
}

\author{
Philippe T. Pinard ${ }^{1}$, Simon Burgess ${ }^{1}$, Anthony Hyde ${ }^{1}$, James Holland ${ }^{1}$ and Peter Statham ${ }^{1}$ \\ ${ }^{1 .}$ Oxford Instruments NanoAnalysis, High Wycombe, UK.
}

A key characteristic of scanning electron microscopes is the ability to characterize micro- to nanometer features scattered over milli- to centimeter-sized specimens. A typical workflow usually consists in navigating over the specimen using the secondary (SE) or backscatter electron (BSE) image in fast scanning mode to locate an interesting region. At this location, the user may decide to store the position for a later analysis or slow down the scan rate to improve the signal-to-noise $(\mathrm{S} / \mathrm{N})$ ratio and/or acquire $\mathrm{X}$-ray spectra, line scans or maps to identify the elements and their concentrations. These steps are often repeated to cover a significant portion of the specimen. The problem with this workflow is that the electron image provides limited and sometimes ambiguous information about the analyzed region. The SE signal shows up topography and the BSE signal can indicate average atomic number but neither signal provides specific chemical information. Knowing the chemical elements is often critical to finding regions of interest and discovering unexpected features. Misinterpretation of the SE and BSE image leads to inefficiency and potentially important, characteristic regions being overlooked.

With the advancement in energy dispersive (ED) spectrometers to achieve large solid angles and electronics capable of operating at high count rates, this sequential workflow can now be changed to a parallel workflow where electron and X-ray images are acquired simultaneously. Improving navigation efficiency however requires more than showing different signals on the same display and three key technologies have been developed to address the barriers to efficient sample exploration.

First, the S/N ratio of single-frame X-ray images is usually much worse than equivalent SE/BSE images, even with large detectors. Combatting this by slowing the scanning rate or reducing the image resolution would slow down the survey or reduce the chance of spotting small features. Instead we have implemented a seamless transition between dynamic and integration modes. While the user is altering the field of view to navigate around a sample, the electron and X-ray images are continuously refreshed so that the user sees dynamic feedback about the topography and chemical distribution in real time. If the user catches sight of a feature of interest and pauses on a field of view, the integration mode is immediately switched on and each new frame of data is added to the previous ones, thus increasing the $\mathrm{S} / \mathrm{N}$ and revealing finer details of the analysed location. As soon as the user decides to move away from the field, the continuous refresh mode is re-instated to provide the fastest response during navigation.

Secondly, it is not enough to collect a large amount of X-rays, the critical part is to accurately identify the chemical elements in the field of view. At high count rates, pile-up events are likely to occur resulting in sum peaks which may be incorrectly identified as elements. While sum peaks can be corrected in spectrum acquired from regions with a homogeneous composition, the same correction on regions containing several phases will probably create artefacts (Figure 1a). By dividing an X-ray map into regions with similar compositions using a clustering algorithm [1], the sum spectrum of each region can be accurately pulse pile-up corrected and the elements can be correctly identified [2]. The recombination of all corrected spectra (Figure 1b) yields a more accurate result and also improved detection of elements only present in small regions. 
Thirdly, when the user is navigating over a large specimen, it may become difficult to assess whether an area of a specimen was investigated. A solution is to store all locations visited during the navigation together with the identified elements and their concentrations so that a visual representation of the elemental distribution over large areas of the sample can be constructed. This can also be combined with a montage of low magnification electron images, as shown in Figure 2.

These three technologies combined with new ED spectrometers enable real-time chemical imaging where complementary information from electron and X-ray images provide a detailed characterization of the analyzed samples.

References:

[1] P Statham et al, Microsc. Microanal. 19 S2 (2013), p. 752.

[2] P Statham, Microsc. Microanal. 19 S2 (2013), p. 1292.

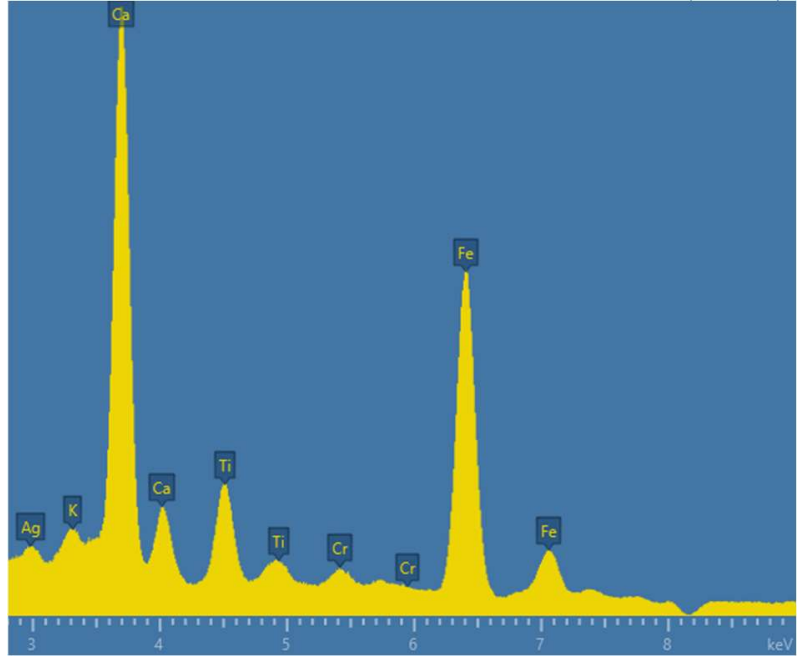

(a)

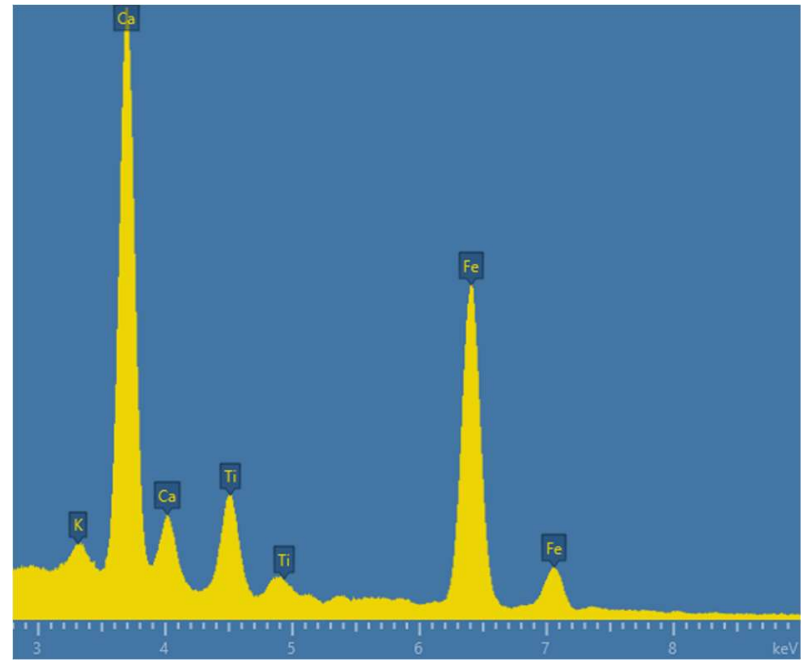

(b)

Figure 1. Sum spectrum of a heterogeneous region with different phases (a) after normal pulse pile-up correction assuming a homogeneous composition and (b) after pulse pile-up correction of individual phases identified using a clustering algorithm. In (a) the sum peak of Si K and $\mathrm{Ca} \mathrm{K}$ is not corrected and $\mathrm{Cr} \mathrm{K}$ is identified. There is also an overcorrection near $8.15 \mathrm{keV}$ resulting in a hole in the spectrum. Both artefacts are removed in (b).

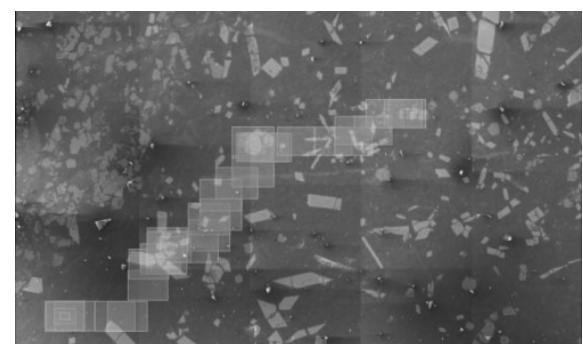

(a)

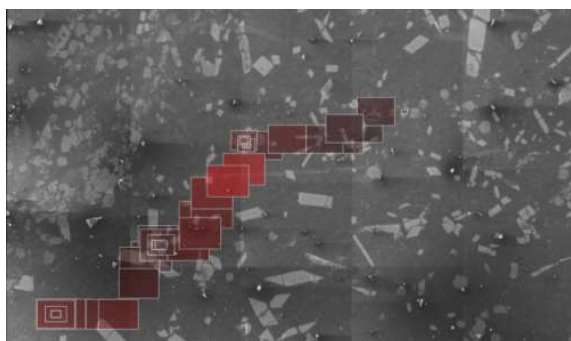

(b)

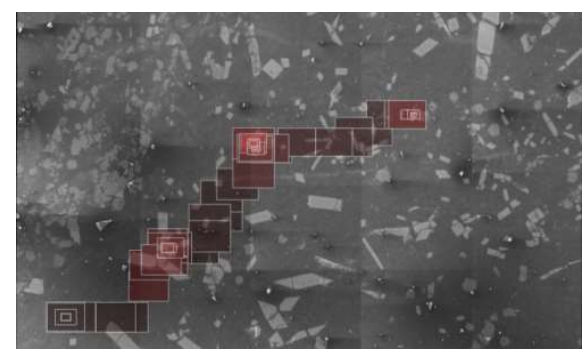

(c)

Figure 2. (a) Trace of all locations investigated during the navigation of a multiphase mineral sample at $20 \mathrm{kV}$. A low magnification secondary electron image is shown in the background and was used for navigation. Regions containing $\mathrm{Ca}$ are highlighted in (b). The intensity of each field of view corresponds to the measure $\mathrm{Ca}$ concentration. Equivalent representation for $\mathrm{Fe}$ in (c). 